\title{
Efecto de la pandemia por COVID-19 en la formación de los residentes de cardiología: más allá del efecto clínico
}

\author{
Impact of COVID-19 pandemic on cardiology fellows academic training: beyond the \\ clinical impact
}

\author{
Yván R. Persia-Paulino ${ }^{1 *}$, Isaac Pascual ${ }^{1,2}$, Rodrigo Fernández-Asensio ${ }^{1}$, Javier Cuevas-Pérez', \\ Antonio Adeba ${ }^{1}$, María Martín ${ }^{1}$, Laura García-Pérez ${ }^{1}$, Pablo Avanzas $^{1,2}$, María Reyes-Cortina ${ }^{3}$, \\ César Moris ${ }^{1,2}$ y José Rozado ${ }^{1}$ \\ ${ }^{1}$ Departamento de Cardiología, Área del Corazón, Hospital Universitario Central de Asturias, Oviedo; ${ }^{2}$ Departamento de Medicina, Universidad de \\ Oviedo, Oviedo; ${ }^{3}$ Departamento de Psiquiatría, Hospital San Agustín, Avilés, Asturias, España
}

\begin{abstract}
Resumen
Objetivo: Determinar la percepción de los médicos internos residentes (MIR) de cardiología de España sobre el efecto de la pandemia por COVID-19 en su formación y la adaptación realizada por sus servicios. Métodos: Estudio de corte transversal a través de una plataforma de encuesta digital con el objetivo de conocer la opinión individual de los MIR de cardiología sobre la influencia de la pandemia en su formación. Se realiza un análisis estadístico para determinar los factores que influyeron en la percepción de la formación afectada. Resultados: Participó un total de 180 MIR de las 17 comunidades autónomas (CA). Los MIR de tercer año fueron los más afectados, junto con los que rotaban en imagen cardíaca. Los residentes de las CA con una prevalencia $>5$ casos/1,000 habitantes fueron los que mayor probabilidad tuvieron de ser desplazados de sus servicios. Conclusiones: Según la opinión de los participantes, el efecto de la pandemia por COVID-19 en su formación fue más negativa en los residentes de tercer año y los que rotaban en imagen cardíaca.
\end{abstract}

Palabras clave: Pandemia. COVID-19. Coronavirus. SARS-CoV-2. Médicos en formación. Formación. Adaptación.

\begin{abstract}
Objective: The objectives were to analyze the perception of the Cardiology Fellows in Training (FIT) of Spain about the impact of the COVID-19 pandemic on their academic training and to know the adaptative changes performed by their department. Methods: A cross-sectional study performed through a digital survey platform for Cardiology FIT. Chi analysis and logistic regression were performed to determine the factors that influenced on the perception of an affected training. Results: $A$ total of 180 FIT from the 17 regions of Spain participated. Third year FIT and those rotating in cardiac imaging were the most affected with statistically significant difference. The residents of the regions with a prevalence of $>5$ cases/1,000 inhabitants were the most likely to be displaced from their departments. Conclusions: According to the opinion of the participants, the impact of the COVID-19 pandemic on their academic training was more negative in third year FITs and those rotating in cardiac imaging.
\end{abstract}

Key words: Pandemic. COVID-19. Coronavirus. SARS-CoV-2. Fellowship. Academic training. Administration and organization.

Correspondencia:

*Yván R. Persia-Paulino

E-mail: yvanpersiap@gmail.com
Disponible en internet: 16-12-2021 Arch Cardiol Mex. 2021;91(Supl):18-24 www.archivoscardiologia.com 1405-9940 / @ 2021 Instituto Nacional de Cardiología Ignacio Chávez. Publicado por Permanyer. Este es un artículo open access bajo la licencia CC BY-NC-ND (http://creativecommons.org/licenses/by-nc-nd/4.0/). 


\section{Introducción}

La COVID-19, causada por el coronavirus SARSCoV-2, ha provocado una pandemia con más de 28.4 millones de casos y 906,000 muertes a nivel mundial hasta el 12 de septiembre del 2020'. España ha sido uno de los países más afectados en Europa ${ }^{2}$ y la adaptación del sistema sanitario español ha jugado un papel clave en la atención de la pandemia. Tal y como ha ocurrido en los hospitales de otros países del mundo ${ }^{3}$, se ha desplazado a los médicos de distintas especialidades de su departamento habitual de trabajo para atender a pacientes afectados por la COVID-19.

Las investigaciones previas han señalado que la adaptación de los sistemas sanitarios a la pandemia por la COVID-19 (suspensión de rotaciones formativas intrahospitalarias, sesiones de docencia y procedimientos no urgentes) podría afectar a la formación de los médicos residentes en formación ${ }^{4}$.

En España, la formación sanitaria especializada se organiza a través del programa médico interno residente (MIR). Después de terminar la escuela de medicina, los médicos graduados deben pasar por una prueba nacional que permite ingresar a un programa de formación, una prueba que es la misma para las especialidades clínicas, quirúrgicas y de medicina familiar. El MIR de cardiología en España es un programa de cinco años de duración, en cuyo primer año se realizan rotaciones clínicas externas no cardiológicas (como medicina interna, nefrología, neumología, endocrinología y otros departamentos afines). El segundo año se destina sobre todo a la cardiología clínica y el tercer año a los cuidados agudos cardiológicos e imagen cardíaca. El cuarto año se compone de modo predominante de hemodinámica y electrofisiología, mientras que los MIR del quinto año rotan principalmente por insuficiencia cardíaca avanzada (incluido el trasplante de corazón y dispositivos de asistencia mecánica) y rotaciones externas específicas de cardiología.

Dentro del ámbito de la cardiología, la actividad asistencial sufrió cambios drásticos durante esos meses ${ }^{5}$. Los médicos internos residentes (MIR) no quedaron exentos de ellos y sus calendarios formativos se han adaptado como consecuencia de esta situación. Sin embargo, se desconoce la transcendencia a largo plazo de estos ajustes, así como la relevancia concebida por los propios residentes. Esta investigación tiene como objetivo conocer la opinión de los MIR de cardiología de España sobre el efecto de la pandemia por
COVID-19 en su formación, así como también los principales cambios organizativos de sus servicios que podrían influir en su formación como cardiólogos.

\section{Métodos}

\section{Diseño del estudio}

Se realizó un estudio de corte transversal mediante el empleo de una encuesta digital, voluntaria y anónima, difundida a través de los correos electrónicos de las distintas comisiones de docencia o secretarías de cardiología de todos los hospitales de España que cuentan con programa de formación sanitaria especializada en cardiología. En el correo electrónico se solicitaba la difusión a los MIR de cardiología de su respectivo hospital, por lo que los investigadores nunca obtuvieron información personal que permitiera identificar o contactar directamente a los participantes. Para llevar a cabo este estudio se obtuvo aprobación del Comité de ética del Principado de Asturias (número de referencia 2020.363). Se solicitó consentimiento informado de manera implícita dentro de la encuesta por la naturaleza del estudio: sin contactar de modo directo con los participantes, anónimo y voluntario.

En la encuesta se indagaba sobre cambios adaptativos en los servicios de cardiología, cambios en rotaciones formativas estipuladas (p. ej., suspensión de rotaciones clínicas, de imagen, intervencionistas, etc.), traslados a otros servicios o centros sanitarios y sobre la percepción en el efecto formativo y emocional. Las preguntas fueron en la modalidad de selección múltiple, con oportunidad de seleccionar sólo una opción por pregunta. El período de encuesta fue del 12 al 21 de mayo del 2020.

\section{Datos epidemiológicos}

Se utilizaron los datos de la Dirección General de Salud Pública, Calidad e Innovación del Ministerio de Sanidad de España, en su actualización número 103 con fecha del 12 de mayo de $2020^{6}$ y datos demográficos del Instituto Nacional de Estadística, que coincidieron con la fecha de inicio del período de encuesta. De estas fuentes se obtuvieron el número de casos totales, la prevalencia (número de casos por cada 1,000 habitantes) y la incidencia en cada una de las 17 comunidades autónomas (CA) de España (en este país, las distintas regiones reciben el nombre de comunidades autónomas). Las CA se clasificaron con 
posterioridad con base en una prevalencia mayor o menor de 5 casos $/ 1,000$ habitantes. Las ciudades autónomas de Ceuta y Melilla no se incluyeron ya que no cuentan con programa formativo de MIR en cardiología.

\section{Análisis estadístico}

El análisis estadístico se realizó con el software STATA 15.2 (Stata Corp. LP, EE.UU.). De manera inicial se realizó un análisis descriptivo de las distintas variables (todas ellas categóricas) para conocer la opinión global de los MIR de cardiología y el porcentaje que consideraba afectada su formación. Después se realizaron comparaciones entre los distintos grupos mediante la prueba estadística ji cuadrada de Pearson. Se realizó un modelo de regresión logística para analizar los factores estudiados que se relacionaron con una percepción negativa en su formación. Por último, se analizaron los cambios organizativos que efectuaron los servicios de cardiología de cada centro y por CA de acuerdo con su prevalencia de casos de COVID-19.

\section{Resultados}

\section{Población estudiada}

Participó un total de 180 MIR de cardiología, con una distribución equitativa de acuerdo con el año de residencia. Se obtuvo la participación de al menos un residente de cada CA, y las CA con mayor representación fueron Andalucía, Madrid y Cataluña. Las características generales de los participantes se reúnen en la Tabla 1.

\section{Adaptación de los programas formativos de los MIR de cardiología}

La rotación formativa del $84 \%(n=151)$ de los encuestados se suspendió, con el $49 \%(n=84)$ que fue desplazado a otro servicio distinto de cardiología. Sólo el $3 \%(n=5)$ se desplazó a otro centro sanitario. El $65 \%(n=118)$ interrumpió alguna rotación por más de un mes y en más de la mitad de los casos (54\%, $n=101)$ la rotación se dio por completada y no recuperable. Los residentes de tercer año son los que podrán recuperar con más frecuencia las rotaciones afectadas, con una diferencia estadísticamente significativa $(p=0.011)$.
Tabla 1. Distribución de frecuencias y porcentajes dentro de la muestra de las respuestas de los MIR de cardiología participantes

\begin{tabular}{|c|c|c|} 
Variable & $\begin{array}{c}\text { Número } \\
\text { participantes } \\
(\mathbf{n}=180)\end{array}$ & $\begin{array}{c}\text { Porcentaje } \\
(\%)\end{array}$ \\
\hline
\end{tabular}

Año de residencia:

- Residente de primer año

- Residente de segundo año

- Residente de tercer año

26.1

- Residente de cuarto año

14.4

- Residente de quinto año

Comunidad Autónoma

- Andalucía

- Aragón

- Asturias, Principado

- Baleares, Islas

- Canarias, Islas

- Cantabria

- Castilla la Mancha

- Castilla y León

- Cataluña

- Comunidad Valenciana

- Extremadura

- Galicia

- Madrid

- Murcia

- Navarra

- País Vasco

- La Rioja

Rotación suspendida

- No

- Sí

15.6

Desplazamiento del servicio

- No

50.6

- Sí

Duración suspensión de rotación

$-<15$ días

- 15-30 días

$-1-2$ meses

$->2$ meses

Rotación suspendida

- Clínica

- Imagen cardíaca

27.2

- Intervencionista

22.2

- Externa/libre

- Otras/ninguna

11.7

28.3

10.6

Solución por parte docencia/ tutor

- Rotación no recuperable

- Recuperación total

- Recuperación parcial

101

56.1

Percepción formación afectad

- Sí, de forma no recuperable

- Sí, de forma leve

- No afecta mi formación

- No, ha enriquecido mi formación
13.3

30.6

9.4

42.8

12.2

35.6 


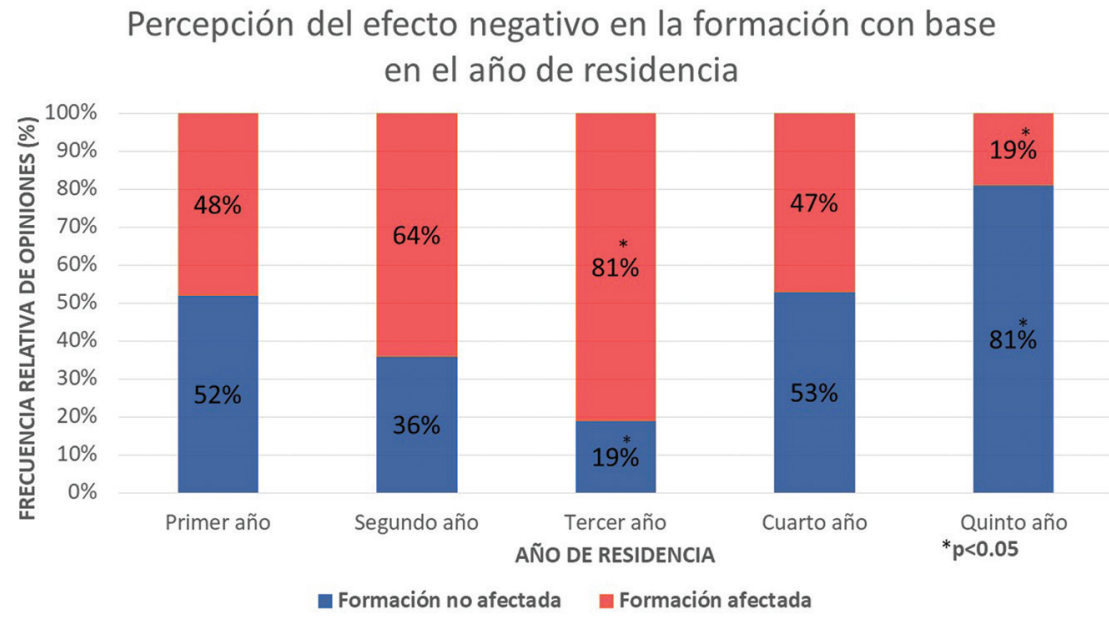

Figura 1. Percepción de los MIR de cardiología de una posible afectación de su formación: con base en el año de residencia en el que se encuentran.

\section{Factores relacionados con la percepción de la influencia negativa en su formación}

Del total de los 180 participantes, el $52 \%(n=94)$ consideró que su formación como especialista se había afectado de manera negativa, aunque el $82 \%(n=77)$ de éstos refiere que fue de forma leve y potencialmente recuperable (Tabla 1). La percepción de los participantes según el año de residencia se observa en la Figura 1. Por el contrario, un $36 \%(n=64)$ de residentes considera que su formación como especialista se ha enriquecido por la pandemia, ya que obligó a trabajar en nuevas áreas en las que no se habría entrenado si no fuera por la situación especial de la pandemia por la COVID-19.

En la regresión logística, los factores relacionados con percibir su formación como afectada negativamente fueron: encontrarse en el tercer año de residencia (OR: 3.66; IC 95\%, 1.013-13.217; $p=0.048$, al compararse con los del primer año de residencia), desplazamiento por más de un mes de su rotación formativa (OR: 7.01; IC 95\%, 1.54-31.99; $p=0.012$ ) y encontrarse en la rotación formativa de imagen cardíaca $(p=0.001)$ (Tabla 2 y Fig. 2). Hallarse en el quinto (último) año de residencia se reveló como un factor protector para percibir su formación como afectada (OR: 0.11; IC 95\%, 0.03-0.48; $p=0.003$, al compararse con los del primer año de residencia).

Con respecto al efecto emocional, el $41 \%(n=74)$ de los participantes considera que, si bien ha vivido momentos estresantes, éstos no influirán en su futuro profesional y sólo el 9\% $(n=17)$ sí considera que tendrá transcendencia a largo plazo.

\section{Cambios organizativos en los servicios de cardiología como resultado de la pandemia por la COVID-19}

En los hospitales, de un $7.22 \%(n=13)$ de los participantes, las guardias de cardiología se suspendieron de manera parcial o total y un $32.22 \%(n=58)$ redujo el número de integrantes de la guardia respecto de lo usual. Si se analiza por año de residencia, no hubo diferencia estadísticamente significativa para ser desplazados del servicio $(p=0.272) 0$ del hospital $(p=0.067)$.

Si se tiene en cuenta la prevalencia de la enfermedad en cada CA, y se compara a los residentes de aquéllas con más de 5 casos/1,000 habitantes (Castilla la Mancha, Castilla León, Cataluña, Comunidad de Madrid, Navarra, País Vasco y La Rioja) en relación con las de menor prevalencia, se identifican diferencias estadísticamente significativas en el desplazamiento de servicio (Fig. $3, p=0.024$ ), pero no en el desplazamiento del hospital $(p=0.064)$ ni en la pérdida/disminución de personal de guardia ( $p=0.638$ ).

\section{Discusión}

El estudio presentado es el primero realizado en España cuyo objetivo es conocer el efecto de la pandemia por COVID-19 en la formación de los MIR. En opinión 


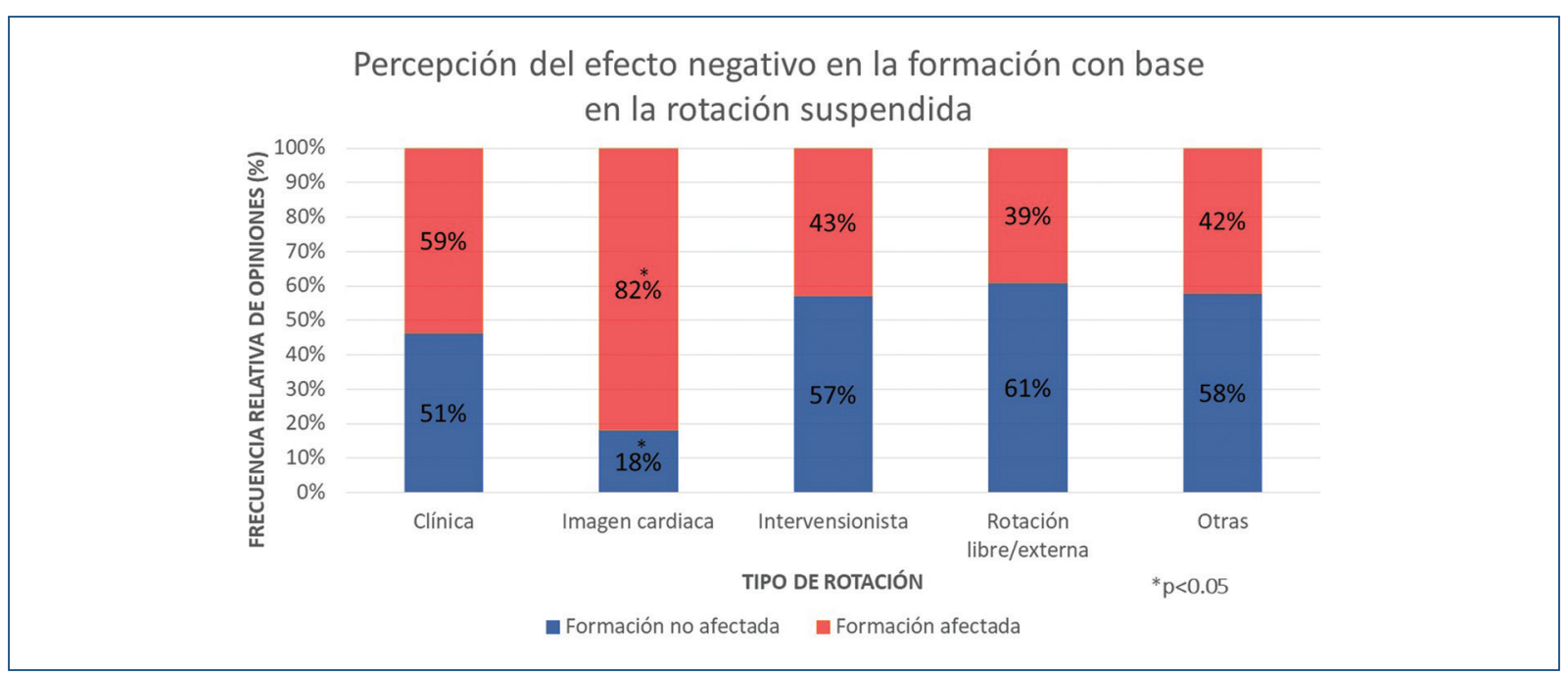

Figura 2. Percepción de los MIR de cardiología de una posible afectación de su formación, de acuerdo con el tipo de rotación suspendida durante la pandemia COVID19. La rotación de imagen cardíaca muestra una diferencia estadísticamente significativa con una $\mathrm{p}<0.001$.

Tabla 2. Regresión logística de la influencia de las principales variables estudiadas y la percepción de una formación afectada.

\begin{tabular}{|c|c|c|c|}
\hline Variables & $\mathbf{R M}$ & $\begin{array}{l}\text { Intervalo de } \\
\text { confianza }\end{array}$ & p \\
\hline $\begin{array}{l}\text { Año de residencia } \\
\text { Residente de segundo año } \\
\text { Residente de tercer año } \\
\text { Residente de cuarto año } \\
\text { Residente de quinto año }\end{array}$ & $\begin{array}{l}1.52 \\
3.66 \\
0.74 \\
0.11\end{array}$ & $\begin{array}{c}0.59-3.94 \\
1.01-13.22 \\
0.24-2.31 \\
0.03-0.48\end{array}$ & $\begin{array}{c}0.39 \\
0.048^{*} \\
0.608 \\
0.003^{*}\end{array}$ \\
\hline $\begin{array}{l}\text { Tiempo de rotación afectado } \\
\text { 15-30 días } \\
\text { 1-2 meses } \\
>2 \text { meses }\end{array}$ & $\begin{array}{c}3.22 \\
7.01 \\
10.67\end{array}$ & $\begin{array}{l}0.70-14.08 \\
1.54-31.99 \\
1.77-64.36\end{array}$ & $\begin{array}{c}0.133 \\
0.012^{*} \\
0.010^{*}\end{array}$ \\
\hline Rotación suspendida & 0.51 & $0.14-1.90$ & 0.314 \\
\hline Desplazamiento del servicio & 1.03 & $0.47-2.22$ & 0.95 \\
\hline Incorporación de otro centro & 0.52 & $0.24-5.25$ & 0.45 \\
\hline Modificación de guardias & 0.99 & $0.24-3.98$ & 0.983 \\
\hline
\end{tabular}

de los residentes de cardiología que participaron, la pandemia ha supuesto cambios sustanciales en su programa formativo y la organización asistencial de sus servicios. Ha representado la pérdida de rotaciones específicas, desplazamientos a otros servicios dentro de su hospital y cambios en la organización de sus servicios.
El porcentaje de MIR en cardiología de esta investigación que percibieron su formación como afectada $(52 \%, n=94)$ resultó similar a la opinión de los directores de programa de centros sanitarios en la ciudad de Nueva York con médicos en formación en cardiología intervencionista, quienes opinaron que la formación de sus médicos en formación se vería moderadamente afectada (57\%) e incluso muy afectada $(14 \%)^{7}$.

La percepción de los MIR, según lo mostrado en los resultados, ha estado condicionada al menos en parte por la situación particular del residente, sobre todo por el año de formación en que cursaba y por la rotación formativa en la que se encontraba. Los residentes que se hallaban a mitad del programa formativo (residentes de tercer año) circulan por rotaciones básicas para la formación de los cardiólogos (como imagen cardíaca y cuidados agudos cardiológicos), lo cual podría ser una de las razones por lo que este grupo de residentes percibió, con una diferencia estadísticamente significativa, que su formación podría afectarse por los meses de rotación que se alteraron. Por otro lado, los residentes de quinto año, quienes ya han superados las rotaciones formativas básicas de la cardiología y se encuentran en rotaciones más específicas (tanto externas como en la parte de insuficiencia cardíaca avanzada) consideraron, también con una diferencia estadísticamente significativa, que su formación como cardiólogos no se afectó por la pandemia, por lo que desde el punto de vista de los autores, cursar el quinto 


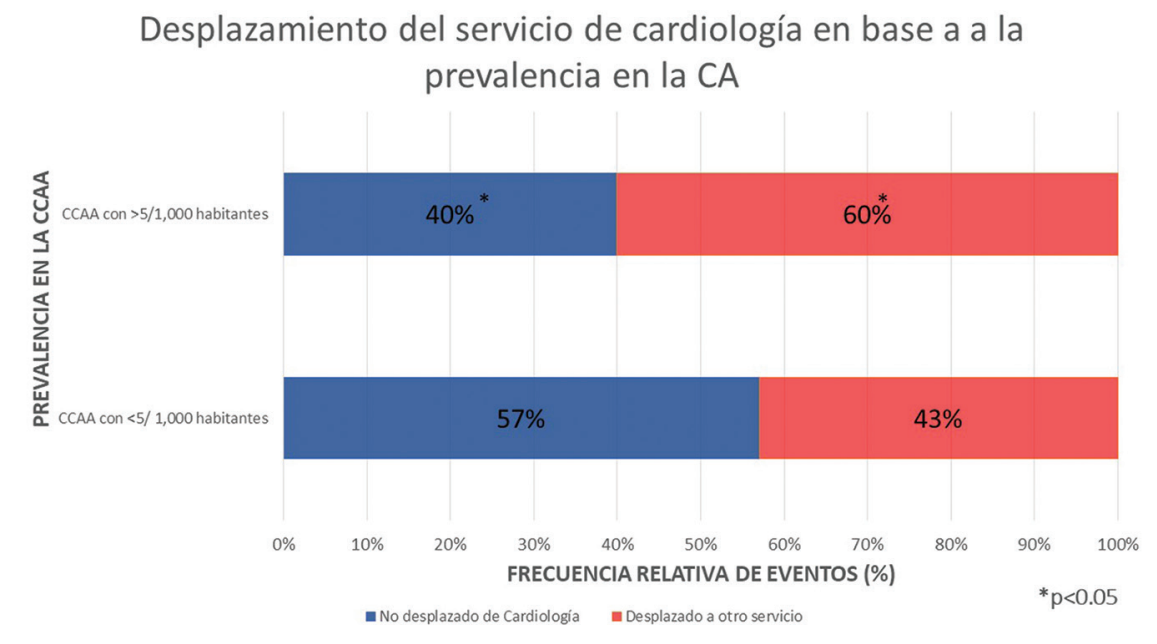

Figura 3. Desplazamiento del servicio de acuerdo con la prevalencia en la CA. Se advierte que el desplazamiento fue menos frecuente en las CA con prevalencia $<5$ casos $/ 1,000$ habitantes, con una $p=0.024$.

MIR: médico interno residente; CA: comunidades autónomas.

año de residencia podría considerarse entonces como un factor "protector".

Se identificaron diferencias de acuerdo con la CA y el mayor desplazamiento del servicio, siendo mayor el desplazamiento en las CA con prevalencia de casos de COVID-19 > 5 casos/1,000 habitantes. Esto puede explicarse porque las CA con mayor prevalencia de casos fueron forzadas quizá a desplazar a sus residentes de sus rotaciones formativas en cardiología a otros lugares de mayor presión asistencial durante la pandemia, similar a lo que ocurrió en otros continentes ${ }^{8}$.

No obstante, en la mayor parte de los casos, la percepción del residente es que esta situación de pandemia por COVID-19 no implicará un detrimento considerable en su formación global, e incluso algunos consideran que ha propiciado un enriquecimiento personal y laboral. El efecto psicológico en los encuestados ha sido menor, de modo semejante a lo observado en médicos residentes de otros países ${ }^{9}$.

Este estudio posee algunas fortalezas, como ser el primer estudio realizado en España cuyo fin es conocer la percepción de los MIR sobre el efecto de la pandemia por la COVID-19 en su formación como médicos especialistas en cardiología. De igual manera, el estudio se condujo de una manera conveniente para la situación epidemiológica crítica vivida en España durante la pandemia, ya que se realizó a través de una plataforma digital (para evitar el contacto personal). Por otro lado, este estudio tiene como limitaciones que no obtuvo la participación de más del $50 \%$ de los MIR en cardiología en el momento de la encuesta, lo que podría limitar la extrapolación de los resultados. El sesgo de selección pudo ser una de las limitantes del estudio, si se considera que la recepción final del correo con el enlace a la encuesta dependió de que el mensaje original se reenviara por las comisiones de docencia/secretaría a los MIR en cardiología para su participación.

En definitiva, este estudio es de interés para conocer cómo la pandemia por la COVID-19 ha influido en la formación de los MIR en cardiología de España. Como perspectiva futura sería de interés desarrollar formas de docencia o simulación que se acoplaran a los programas formativos actuales y sirvieran para situaciones en las que los médicos residentes de distintas especialidades no puedan llevar a cabo un número adecuado de intervenciones o procedimientos por situaciones ajenas al sistema de salud, como otra pandemia. Sería conveniente también conocer la percepción sobre el efecto de la pandemia en la formación de otros médicos en formación (tanto de grado como de especialización en alguna rama de la medicina) en el resto de Europa, América y el mundo.

\section{Conclusión}

La pandemia por la COVID-19 ha llevado a la reorganización de la actividad asistencial, en casi todos los casos con afectación directa del programa formativo de los MIR en cardiología de España. Si bien la mayoría de los MIR en cardiología suspendió de manera 
transitoria su programa formativo, la percepción negativa en su formación fue mayor en los residentes que se encontraban a mitad del período formativo y quienes rotaban en imagen cardíaca.

\section{Financiamiento}

Ninguno.

\section{Conflicto de intereses}

Los autores declaran que no tienen conflicto de intereses con la autoría o publicación de este artículo.

\section{Aprobación ética}

Se obtuvo aprobación por parte del Comité de ética del Principado de Asturias, con número de referencia 2020.363.

\section{Responsabilidades éticas}

Protección de sujetos humanos y animales. Los autores declaran que no se realizaron experimentos en seres humanos 0 animales para este estudio.

Confidencialidad de los datos. Los autores declaran haber seguido los protocolos de su centro de trabajo sobre la publicación de datos de pacientes.
Derecho a la privacidad y consentimiento informado. Los autores han obtenido el consentimiento informado por escrito de los pacientes o sujetos mencionados en el artículo. El autor de correspondencia se halla en posesión de este documento.

\section{Bibliografía}

1. World Health Organization. Coronavirus disease (COVID-19) outbreak situation, https://www.who.int/emergencies/diseases/novel-coronavirus-2019? gclid=Cj0KCQjwuJz3BRDTARIsAMg-HxV04JjTLj_io4Uw1EmcV_HZOPvsZX7ucBhZh8_MpjIMOOCwE4JxoPcaAgmSEALw_wcB; 2020. Accessed in september 12 of 2020.

2. Ceylan Z. Estimation of COVID-19 prevalence in Italy, Spain, and France. Science of The Total Environment. 2020;729(138817):1-7. Available from: http://dx.doi.org/10.1016/j.scitotenv.2020.138817

3. Ammar A, Stock AD, Holland R. Managing a specialty service during the COVID-19 Crisis. Academic Medicine. 2020;Publish Ahead of Print. Available from: http://dx.doi.org/10.1097/ACM.0000000000003440

4. Liang ZC, Ooi SBS, Wang W. Pandemics and their impact on medical training: lessons from Singapore. Academic Medicine. 2020;95(9):135961. Available from: http://dx.doi.org/10.1097/ACM.0000000000003441

5. DeFilippis EM, Stefanescu AC, Reza N. Adapting the educational environment for cardiovascular fellows-in-training during the COVID-19 Pandemic. J Am Coll Cardiol. 2020;75(20):2630-4. Available from: https://doi. org/10.1016/j.jacc.2020.04.013

6. Ministerio de Sanidad de Espana. Actualizacion no 103. Enfermedad por el coronavirus (COVID-19). https://www.lamoncloa.gob.es/serviciosdeprensa/notasprensa/sanidad14/Documents/2020/12052020_Actualizacion103COVID19.pdf; 2020. Accessed in may 12 of 2020.

7. Gupta T, Nazif TM, Vahl TP. Impact of the COVID-19 pandemic on interventional cardiology fellowship training in the New York metropolitan area: a perspective from the United States epicenter. Catheter Cardiovasc Interv. 2020;(May):1-5. DOI: 10.1002/ccd.28977

8. Kee A, Archuleta S, Dan YY. Internal medicine residency training in the COVID-19 era-reflections from Singapore. Journal of Graduate Medical Education. 2020;12(4):406-408. doi:10.4300/jgme-d-20-00315.1

9. Zhang Y, Ma ZF. Impact of the COVID-19 pandemic on mental health and quality of life among residents in Liaoning Province, China: a cross-sectional study. Int J Environ Res Public Health. 2020;17:1-12. Available form: https://doi.org/10.3390/ijerph17072381 